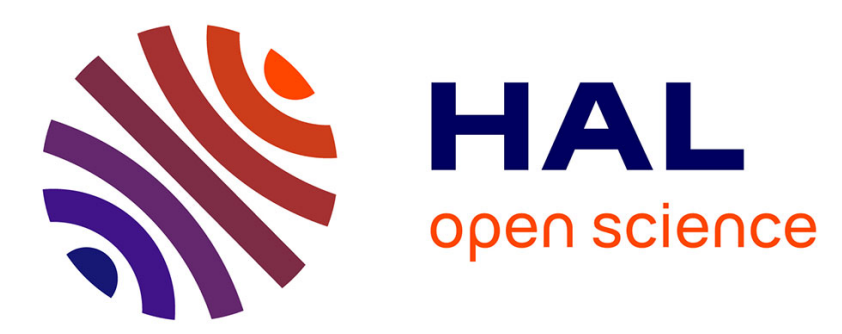

\title{
Event-triggered tracking control of unicycle mobile robots
}

\author{
Romain Postoyan, Marcos Cesar Bragagnolo, Ernest Galbrun, Jamal Daafouz, \\ Dragan Nesic, Eugênio Castelan
}

\section{- To cite this version:}

Romain Postoyan, Marcos Cesar Bragagnolo, Ernest Galbrun, Jamal Daafouz, Dragan Nesic, et al.. Event-triggered tracking control of unicycle mobile robots. Automatica, 2015, 52, pp.302-308. 10.1016/j.automatica.2014.12.009 . hal-01099190

\section{HAL Id: hal-01099190 https://hal.science/hal-01099190}

Submitted on 31 Dec 2014

HAL is a multi-disciplinary open access archive for the deposit and dissemination of scientific research documents, whether they are published or not. The documents may come from teaching and research institutions in France or abroad, or from public or private research centers.
L'archive ouverte pluridisciplinaire HAL, est destinée au dépôt et à la diffusion de documents scientifiques de niveau recherche, publiés ou non, émanant des établissements d'enseignement et de recherche français ou étrangers, des laboratoires publics ou privés. 


\title{
Event-triggered tracking control of unicycle mobile robots
}

\author{
R. Postoyan ${ }^{\text {a M. C. Bragagnolo }}{ }^{\text {aE. Galbrun }}{ }^{\mathrm{a}}$ J. Daafouz ${ }^{\mathrm{a}}$ D. Nešić ${ }^{\mathrm{b}}$ E.B. Castelan ${ }^{\mathrm{c}}$ \\ ${ }^{a}$ Université de Lorraine, CRAN, UMR 7039 and CNRS, CRAN, UMR 7039, France \\ ${ }^{\mathrm{b}}$ Department of Electrical and Electronic Engineering, the University of Melbourne, Parkville, VIC 3010, Australia \\ ${ }^{\mathrm{c}}$ Grupo de Controle de Sistemas Mecatronicos - CSM Depto. de Automação e Sistemas - DAS Universidade Federal de Santa \\ Catarina - UFSC Florianópolis, Brazil
}

\begin{abstract}
We investigate the stabilization of time-varying trajectories for unicycle mobile robots using event-triggered controllers. We follow an emulation-like approach in the sense that we first synthesize the controller while ignoring the communication constraints and we then derive an appropriate triggering condition. The solutions to the robot model are proved to practically converge towards the given reference trajectory, under some condition on the latter. Furthermore, the existence of a uniform minimum amount of times between any two transmissions is ensured. Afterwards, experimental results are presented where the controller has been implemented on a remote computer which transmits its output to the mobile robot via a IEEE 802.11g wireless network. The proposed event-triggering strategy is able to significantly reduce the need for communication compared to a classical time-triggered setup while ensuring similar, if not better, tracking performances.
\end{abstract}

Key words: Event-triggered control, hybrid systems, nonholonomic systems, nonlinear systems.

\section{Introduction}

The objective of the paper is to guarantee the tracking of given reference trajectories by unicycle mobile robots using a remote controller, while reducing the usage of the communication channel. In particular, we want to limit the amount of control input updates to save communication resources and to reduce the risk of packet losses and long transmission delays; noting that that also allows to curb the actuators wear and to reduce the energy consumption of the actuators. An event-triggered

\footnotetext{
ऋ A preliminary version of this paper was presented at the $9^{\text {th }}$ IFAC Symposium on Nonlinear Control Systems [11]. This work was supported by the ANR under the grant COMPACS (ANR-13- BS03-0004-02), the European 7th Framework Network of Excellence Highly-complex and networked control systems (HYCON2 No. 257462) and the Australian Research Council under the Discovery Projects and Future Fellowship schemes. Corresponding author R. Postoyan. Tel. $+33(0) 3.83 .59 .56 .47$.

Email addresses: romain.postoyan@univ-lorraine.fr (R. Postoyan),

marcos-cesar bragagnolo@univ-lorraine.fr (M. C. Bragagnolo), ernest.galbrun@univ-lorraine.fr (E. Galbrun), jamal.daafouz@univ-lorraine.fr (J. Daafouz), dnesic@unimelb.edu.au (D. Nešić),

eugenio.castelan@das.ufsc.br (E.B. Castelan).
}

feedback law is designed for that purpose. The idea of event-triggered control is to transmit data between the controller and the plant whenever a state-dependent criterion is satisfied and not periodically as in traditional setups. In that way, the transmissions are adapted to the state of the system and these only occur when it is needed. Various techniques have been developed, see $[1,2,7,12,16]$ to mention a few. Most of them address the stabilization of equilibrium points, while very few controllers have been synthesized to stabilize time-varying trajectories, see e.g., [17]. It appears that tracking control induces additional difficulties as only approximate tracking can usually be ensured under communication constraints because of the time-varying component of the control law, see for more detail [13]. As a consequence, available results on the stabilization of equilibrium points are not directly applicable in this context.

We follow an emulation-like approach (see e.g., $[16,12]$ ). Thus, we first design the controller while ignoring the communication constraints and we derive the triggering strategy afterwards. We have selected the state-feedback controller of [8] among others, because the law is continuous and an explicit Lyapunov function is provided. The continuity of the feedback law is useful to guarantee the existence of a minimum amount of time between two transmissions, which is essential in practice as the hard- 
ware cannot transmit infinitely fast. The Lyapunov function is used to design the event-triggering condition. This Lyapunov function is weak in the sense that it does not strictly decrease along the solutions of the closed-loop system in the absence of communication constraints, see [8]. To overcome this issue, we impose conditions on the reference trajectories under which the Lyapunov function strictly decreases outside a given neighborhood of the origin, which can be made as small as desired by appropriately tuning the control law parameters. We then take into account the effects of the network and we model the problem as a hybrid system using the formalism of [5] (as in e.g., $[3,12,15]$ ). The event-triggering condition we construct is inspired by the technique in $[16,4]$ where the Lyapunov function is forced to decrease at a certain rate, except in the aforementioned neighborhood of the origin, which we call a dead-zone. We prove that the solutions of the robot model practically converge towards the reference trajectories. The ultimate bound depends on a tuneable parameter which corresponds to the 'size' of the dead-zone of the triggering condition. It can be used to adjust the accuracy of the tracking error at the price of more transmissions. The approach we follow is similar to the one in [17], however we do not impose the same conditions on the reference trajectories and we propose a different triggering law. Finally, we have implemented the event-triggered controller on a benchmark. The controller sends its data to the robot over a wireless network according to the triggering condition and the measurements are periodically collected by cameras which are directly connected to the remote controller via a dedicated high bandwidth wired channel. The experimental results show that it is possible to significantly reduce the usage of the wireless network compared to periodic sampling, if we agree to slightly give up on the tracking accuracy.

A similar problem is investigated in [14] where selftriggered controllers are developed. Beside the fact that we consider a nonlinear model of the robot and not a linear model as in [14], we also envision a different setup. In our case, no local controller is implemented on the robot which helps saving batteries and the measurements are given by cameras which are connected via a wired channel to the controller unit (as opposed to odometry in [14]), which justifies the choice of an eventtriggered implementation. Compared to our preliminary work in [11], the results rely on a simplified stability analysis and on different assumptions on the reference trajectories which cover a class of well-motivated cases. In particular, we are able to guarantee the existence of a minimum amount of time between two transmissions for both of the reference trajectories we consider in the experimental part, which is not the case of [11]. Furthermore, we present new experimental results as we use cameras to measure the robot position as explained above and not odometry as in [11]; noting that the latter has the drawback to use the wireless network periodically to communicate with the controller.
The paper is organized as follows. Preliminaries are given in Section 2. In Section 3, the controller of [8] is recalled and the hybrid model is introduced. The event-triggering condition and the analytical results are presented in Section 4 . Section 5 deals with the experimental results. The proofs are provided in the Appendix.

\section{Preliminaries}

Let $\mathbb{R}=(-\infty, \infty), \mathbb{R}_{\geq 0}=[0, \infty), \mathbb{R}_{>0}=(0, \infty), \mathbb{Z}_{\geq 0}=$ $\{0,1,2, \ldots\}$, and $\mathbb{Z}_{>0}=\{1,2, \ldots\}$. For $(x, y) \in \mathbb{R}^{n+m}$, the notation $(x, y)$ stands for $\left[x^{\mathrm{T}}, y^{\mathrm{T}}\right]^{\mathrm{T}}$. A function $\gamma$ : $\mathbb{R}_{\geq 0} \rightarrow \mathbb{R}_{\geq 0}$ is of class $\mathcal{K}$ if it is continuous, zero at zero and strictly increasing, and it is of class $\mathcal{K}_{\infty}$ if in addition it is unbounded. A continuous function $\gamma: \mathbb{R}_{\geq 0}^{2} \longrightarrow \mathbb{R}_{\geq 0}$ is of class $\mathcal{K} \mathcal{L}$ if for each $t \in \mathbb{R}_{>0}, \gamma(\cdot, t)$ is of class $\mathcal{K}$, and, for each $s \in \mathbb{R}_{>0}, \gamma(s, \cdot)$ is decreasing to zero. For a right-continuous function $f: \mathbb{R} \rightarrow \mathbb{R}^{n}, f\left(t^{+}\right)$stands for $\lim _{s \rightarrow t, s>t} f(s)$ for $t \in \mathbb{R}$. We recall that the function sinc is defined from $\mathbb{R}$ to $\mathbb{R}$ by $\operatorname{sinc}(x)=\frac{\sin x}{x}$ when $x \neq 0$, and $\operatorname{sinc}(0)=1$, and it is twice continuously differentiable with $\operatorname{sinc}^{\prime}(0)=0$ and $\operatorname{sinc}^{\prime \prime}(0)=-\frac{1}{3}$.

We will write the event-triggered controlled system as a hybrid system using the formalism of [5]. In particular, we will consider a system of the form

$$
\dot{x}=f(x) x \in C, \quad x^{+}=g(x) x \in D,
$$

where $x \in \mathbb{R}^{n}$ is the state and $C, D \subset \mathbb{R}^{n}$ are respectively the flow and the jump sets. We assume that $f$ and $g$ are continuous and that $C$ and $D$ are closed sets (this will be the case in the paper). We recall some basic definitions, see [5]. A set $E \subset \mathbb{R}_{\geq 0} \times \mathbb{Z}_{\geq 0}$ is a compact hybrid time domain if $E=\underset{j \in\{0, \ldots, J-1\}}{\cup}\left(\left[t_{j}, t_{j+1}\right], j\right)$ for some finite sequence of times $0=t_{0} \leq t_{1} \leq \ldots \leq t_{J}$. The set $E$ is a hybrid time domain if for all $(T, J) \in E, E \cap([0, T] \times$ $\{0,1, \ldots, J\})$ is a compact hybrid time domain. A hybrid arc is a function $\phi$ defined on a hybrid time domain $\operatorname{dom} \phi$ and such that, for each $j \in \mathbb{Z}_{\geq 0}, t \mapsto \phi(t, j)$ is locally absolutely continuous on $I^{j}:=\{t:(t, j) \in$ $\operatorname{dom} \phi\}$. A hybrid arc $\phi: \operatorname{dom} \phi \rightarrow \mathbb{R}^{n}$ is a solution to (1) if: (i) $\phi(0,0) \in C \cup D$; (ii) for all $j \in \mathbb{Z}_{\geq 0}$ and almost all $t \in I^{j}, \phi(t, j) \in C$ and $\dot{\phi}(t, j)=f(\phi(t, j))$; (iii) for $(t, j) \in \operatorname{dom} \phi$ such that $(t, j+1) \in \operatorname{dom} \phi, \phi(t, j) \in D$ and $\phi(t, j+1)=g(\phi(t, j))$. A solution to (1) is said to be: nontrivial if dom $\phi$ contains at least two points; maximal if it cannot be extended; complete if dom $\phi$ is unbounded.

The definition below characterizes hybrid systems that generate solutions for which two successive jumps are spaced by (at least) a strictly positive amount of time which is uniform over the ball of initial conditions (see $[12])$. 


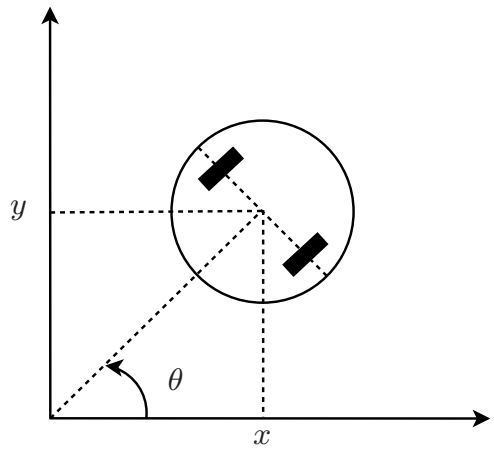

Fig. 1. Mobile robot.

Definition 1 The solutions to (1) have a uniform semiglobal dwell-time if for any $\Delta \geq 0$, there exists $\eta(\Delta)>0$ such that for any solution $\phi$ to (1) with $|\phi(0,0)| \leq \Delta$,

$$
\sup I^{j}-\inf I^{j} \geq \eta(\Delta) \quad \forall j \in \mathbb{Z}_{>0} \text { with } I^{j} \neq \emptyset .
$$

We recall the following invariance definition (see [5]).

Definition 2 Consider system (1), the set $\mathcal{A} \subset \mathbb{R}^{n}$ is strongly pre-forward invariant if for any $\xi \in \mathcal{A}$ each solution $\phi$ with initial condition $\xi$ satisfies $\phi(t, j) \in \mathcal{A}$ for all $(t, j) \in \operatorname{dom} \phi$.

\section{System model}

\subsection{Jiang and Nijmeijer's controller}

We consider a mobile robot for which the dynamics are defined by the following nonholonomic system

$$
\dot{x}=v \cos (\theta), \quad \dot{y}=v \sin (\theta), \quad \dot{\theta}=w,
$$

where $(x, y)$ are the Cartesian coordinates and $\theta$ is the angle between the heading direction and the $x$-axis, $(v, w)$ denotes the control input vector, see Figure 1. The objective is to make the solution to system (3) track a given trajectory $t \mapsto\left(x_{r}(t), y_{r}(t), \theta_{r}(t)\right)$. We restrict our attention to the case where the reference trajectory satisfies the following dynamical equations

$$
\dot{x}_{r}=v_{r} \cos \left(\theta_{r}\right), \quad \dot{y}_{r}=v_{r} \sin \left(\theta_{r}\right), \quad \dot{\theta}_{r}=w_{r},
$$

where $v_{r}$ and $w_{r}$ are the feedforward inputs to system (4). In practice, the term $w_{r}$ is obtained by differentiating $\theta_{r}$. The term $v_{r}$ is deduced by using that $v_{r}^{2}=$ $\left(\dot{x}_{r}\right)^{2}+\left(\dot{y}_{r}\right)^{2}$ and its sign is obtained by investigating the first equations of (4) for the given trajectory $\left(x_{r}, y_{r}, \theta_{r}\right)$.
We refer to (4) as the reference system. We use the following error coordinates (see [9])

$$
\left(\begin{array}{l}
x_{e} \\
y_{e} \\
\theta_{e}
\end{array}\right)=\left(\begin{array}{ccc}
\cos (\theta) & \sin (\theta) & 0 \\
-\sin (\theta) & \cos (\theta) & 0 \\
0 & 0 & 1
\end{array}\right)\left(\begin{array}{l}
x_{r}-x \\
y_{r}-y \\
\theta_{r}-\theta
\end{array}\right)
$$

which leads to the error system

$$
\begin{aligned}
& \dot{x}_{e}=w y_{e}-v+v_{r} \cos \left(\theta_{e}\right), \dot{y}_{e}=-w x_{e}+v_{r} \sin \left(\theta_{e}\right) \\
& \dot{\theta}_{e}=w_{r}-w .
\end{aligned}
$$

The change of coordinates in (5) is invertible and the transformation matrix and its inverse are bounded. As a consequence, if we guarantee that $x_{e}, y_{e}, \theta_{e}$ converge to a neighborhood of the origin, then so do $x_{r}-x, y_{r}-y, \theta_{r}-$ $\theta$. In [8], the following controller is shown to ensure the global convergence of $x_{e}, y_{e}, \theta_{e}$ towards the origin (under some conditions on the feedforward inputs $v_{r}$ and $w_{r}$ )

$$
v=v_{1}+c_{1} \bar{x}_{e}, \quad w=w_{r}+\gamma y_{e} v_{r} \operatorname{sinc} \theta_{e}+c_{2} \gamma \theta_{e},
$$

where $c_{1}, c_{2}, \gamma>0$ are design parameters and

$$
\begin{aligned}
& \bar{x}_{e}:=x_{e}-c_{3} w y_{e} \\
& v_{1}:=v_{r} \cos \theta_{e}-c_{3} \dot{w} y_{e}+c_{3} w\left(w x_{e}-v_{r} \sin \theta_{e}\right)
\end{aligned}
$$

with $c_{3}>0$. We do not necessarily require to differentiate online $\dot{w}$ to compute the term $v_{1}$ in (8) as we can replace $\dot{w}$ by its algebraic expression by using (3), (4) and (7).

\subsection{Assumptions on the feedforward inputs}

We make the following assumption on the feedforward inputs $v_{r}$ and $w_{r}$.

Assumption 3 There exists $M \geq 0$ such that the absolute values of $v_{r}(t), w_{r}(t), \dot{v}_{r}(t)$ and $\dot{w}_{r}(t)$ are bounded by $M \geq 0$ for any $t \in[0, \infty)$. Furthermore there exists $c \in \mathbb{R}_{>0}$ such that $v_{r}(t)^{2} \geq c^{2}$ for any $t \geq 0$.

The first part of Assumption 3 corresponds to the first condition of Proposition 2 in [8]. We also assume that the sign of $v_{r}$ is always the same and that $v_{r}$ does not become arbitrarily close to the origin. The latter differs from $[8,11]$ and is satisfied by various reference trajectories, as those studied in Section 5 for example.

\subsection{Network implementation \&3 hybrid model}

We focus on the scenario where the controller $(v, w)$ in (7) communicates with the robot (3) via a network, see 


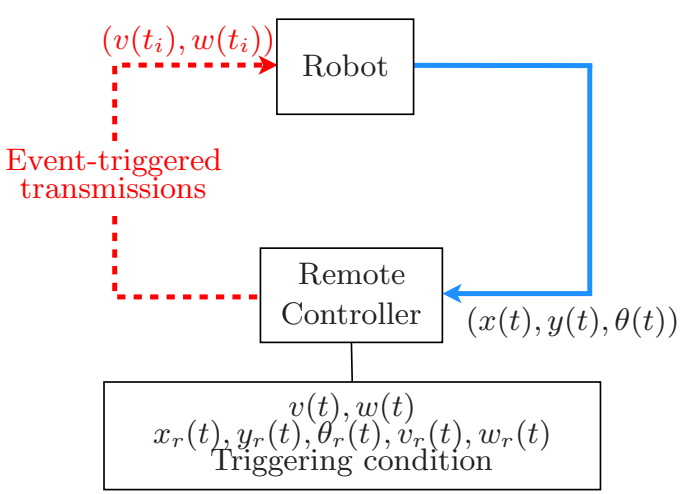

Fig. 2. Controller implementation.

Figure 2. We assume that the controller has access to the reference trajectory and the feedforward inputs $v_{r}$ and $w_{r}$ at any time.

The sequence of transmission instants is $t_{i}, i \in \mathbb{Z}_{\geq 0}$, and it is defined according to a state-dependent criterion which will be specified in Section 4.1. Thus, we have, for all $t \in\left[t_{i}, t_{i+1}\right)$,

$\dot{x}_{e}=\hat{w} y_{e}-\hat{v}+v_{r} \cos \left(\theta_{e}\right), \dot{y}_{e}=-\hat{w} x_{e}+v_{r} \sin \left(\theta_{e}\right)$

$\dot{\theta}_{e}=w_{r}-\hat{w}$

where $\hat{v}$ and $\hat{w}$ are respectively the networked versions of $v$ and $w$. The controller is implemented using zeroorder-hold devices, i.e. $\hat{v}(t)=v\left(t_{i}\right)$ and $\hat{w}(t)=w\left(t_{i}\right)$ for $t \in\left[t_{i}, t_{i+1}\right)$. Hence the controller is, for $i \in \mathbb{Z}_{\geq 0}$,

$$
\left.\begin{array}{l}
\dot{\hat{w}}=0 \\
\dot{\hat{v}}=0
\end{array}\right\} t \in\left[t_{i}, t_{i+1}\right) \quad \begin{aligned}
& \hat{w}\left(t_{i}^{+}\right)=w\left(t_{i}\right) \\
& \hat{v}\left(t_{i}^{+}\right)=v\left(t_{i}\right) .
\end{aligned}
$$

We write the model using the formalism of [5], for which a jump represents a transmission

$$
\dot{q}=f(q) \quad q \in C, \quad q^{+}=g(q) \quad q \in D,
$$

with $q:=\left(x_{e}, y_{e}, \theta_{e}, \hat{w}, \hat{v}, \tau\right)$,

$$
f(q):=\left(\begin{array}{c}
\hat{w} y_{e}-\hat{v}+v_{r} \cos \left(\theta_{e}\right) \\
-\hat{w} x_{e}+v_{r} \sin \left(\theta_{e}\right) \\
w_{r}-\hat{w} \\
0 \\
0 \\
1
\end{array}\right), g(q):=\left(\begin{array}{c}
x_{e} \\
y_{e} \\
\theta_{e} \\
v \\
w \\
\tau
\end{array}\right),
$$

where $\tau$ is the time-variable, which is introduced to render the model autonomous by considering the feedforward inputs as time-dependent terms, and $v, w$ are de- fined in (7). The sets $C$ and $D$ are defined by the triggering condition. Indeed, the set $C$ corresponds to the region of the state-space where the triggering condition is not satisfied and $D$ is defined as the region of the statespace where the triggering condition is verified (which does not mean that $C \cap D=\emptyset$ as we will see). In that way, defining the triggering condition is equivalent to defining the sets $C$ and $D$ (see e.g., [12]). The model (11) is a simplification of the experimental setup considered in Section 5 as we assume that the triggering condition is continuously evaluated in (11). Nevertheless, system (11) is useful to design the event-triggered controller, and we will see that the stability property we ensure for system (11) can be maintained when the sampling of the triggering mechanism is sufficiently small.

Our objective is to design a triggering condition, i.e. the sets $C$ and $D$ in (11), such that the errors $x_{e}, y_{e}, \theta_{e}$ practically converge to the origin. The tuneable parameter will be given by the triggering condition.

\section{Design \& analytical results}

\subsection{Event-triggering condition}

The Lyapunov-like candidate function below is considered in [8]

$$
V(q)=\frac{1}{2} \bar{x}_{e}^{2}+\frac{1}{2} y_{e}^{2}+\frac{1}{2 \gamma} \theta_{e}^{2}
$$

where $\bar{x}_{e}$ is defined in (8) and $\gamma$ comes from (7). We derive, by following similar lines as in [8], that for any $q \in \mathbb{R}^{6}$,

$$
\langle\nabla V(q), f(q)\rangle=-\Sigma(q)+\Lambda(q)
$$

where $\Sigma(q):=c_{1} \bar{x}_{e}^{2}+c_{2} \theta_{e}^{2}+c_{3} w^{2} y_{e}^{2}$ and $\Lambda(q):=$ $\bar{x}_{e}\left(e_{w} y_{e}-e_{v}-c_{3} y_{e} e_{w} \rho\left(x_{e}, y_{e}, \theta_{e}, v_{r}\right)+c_{3} w e_{w} x_{e}\right)-$ $y_{e} e_{w} x_{e}-\frac{1}{\gamma} \theta_{e} e_{w}$, with $\rho\left(x_{e}, y_{e}, \theta_{e}, v_{r}\right):=-\gamma x_{e} v_{r} \operatorname{sinc} \theta_{e}-$ $\gamma y_{e} v_{r} \operatorname{sinc}^{\prime}\left(\theta_{e}\right)+c_{2} \gamma, e_{w}:=\hat{w}-w$ and $e_{v}: \hat{v}-v$. We note that we recover (41) in [8] when there is no network, i.e. when $e_{v}=e_{w}=0(\operatorname{as} \Lambda(q)=0$ in this case $)$.

We define the flow and the jump sets as follows

$$
\left.\begin{array}{l}
C=\left\{q \in \mathcal{Q}:\langle\nabla V(q), f(q)\rangle \leq-\sigma \Sigma(q) \text { or } V(q) \leq \varepsilon^{2}\right\} \\
D=\left\{q \in \mathcal{Q}:\langle\nabla V(q), f(q)\rangle \geq-\sigma \Sigma(q) \text { and } V(q) \geq \varepsilon^{2}\right.
\end{array}\right\}
$$

where $\mathcal{Q}:=\mathbb{R}^{5} \times \mathbb{R}_{>0}, \sigma \in(0,1)$ and $\varepsilon>0$ are design parameters. The idea is the following. In [8], the function $V$ is ensured to decrease with the rate $\Sigma$. Here, we degrade this decreasing property by the factor $\sigma$ so that $V$ still decreases but with rate $\sigma \Sigma$ with $\sigma \in(0,1)$, like in [16]. In addition, we impose that condition only when $V(q) \geq \varepsilon^{2}$ 
as otherwise we can neither guarantee the convergence of $x_{e}, y_{e}, \theta_{e}$ to the origin, nor ensure the existence of a minimum amount of time between two transmission instants under Assumption 3. That is in agreement with [13] where it is shown that the time-triggered emulation of a nonlinear tracking controller leads to approximate tracking when the feedforward inputs are subject to communication constraints, which is the case here, even with very fast sampling. Similar ideas are followed in $[4,12,15]$ to design the triggering condition. Nevertheless, none of the strategies proposed in these papers directly applies to our problem as the triggering condition is based on a weak Lyapunov function $V$ (as opposed to strong Lyapunov functions) and these do not address tracking control.

\subsection{Stability analysis}

We first state the following lemma.

Lemma 4 Suppose Assumption 3 holds, then for any $c_{1}, c_{2}, c_{3}, \varepsilon>0$ and $\gamma>0$ sufficiently large, there exists $\vartheta>0$ such that for any $q \in \mathcal{Q}$

$$
V(q) \geq \varepsilon^{2} \Rightarrow \Sigma(q) \geq \vartheta
$$

When $q \in C$ and $V(q)>\varepsilon^{2}$, we know from (15) that $\langle\nabla V(q), f(q)\rangle \leq-\sigma \Sigma(q)$. Lemma 4 implies that in this case $\langle\nabla V(q), f(q)\rangle \leq-\sigma \vartheta<0$, i.e. that $V$ strictly decreases on this part of the set $C$. That is very useful for the stability analysis as it allows us avoiding invoking hybrid invariance principle as in [11]. Moreover, Lemma 4 also plays a key role to prove the existence of a minimum amount of time between two jumps (see the proof of Theorem 5). We are ready to state the main result of this section.

Theorem 5 Consider system (11) and suppose Assumption 3 holds. For any $c_{1}, c_{2}, c_{3}, \varepsilon>0$ and $\gamma>0$ sufficiently large, the following holds.

(i) There exists $\beta \in \mathcal{K} \mathcal{L}$ such that for any solution $\phi$, $V(\phi(t, j)) \leq \beta(V(\phi(0,0)), t+j)+\varepsilon^{2}$ for any $(t, j) \in$ dom $\phi$.

(ii) The solutions have a uniform semiglobal dwell-time.

(iii) The maximal solutions are complete.

Items (i) and (iii) of Theorem 5 together with the boundedness of $v_{r}$ and $w_{r}$ stated in Assumption 3 ensure that the states of the robot converge to the states of the reference system up to an error of the order of $\varepsilon$. Item (ii) of Theorem 5 satisfies the requirement that any two successive transmissions cannot occur arbitrarily close-intime, which is important in practice.

\subsection{Digital implementation}

As already mentioned, the triggering condition is not continuously evaluated in practice but only periodically ${ }^{1}$ every $T$ units of time, see Section 5 . The sampling effect can be modeled as a time-varying delay $\delta \in[0, T]$ which affects the control inputs. Indeed, the control input applied to the plant is sent at least after $T$ units of time have elapsed since the triggering condition (15) has been violated. The proof of Theorem 5 indicates that in this case, for $T$ sufficiently small, there exists $\sigma^{\prime} \in(0, \sigma)$ such that the triggering condition defined by (15) is satisfied with $\sigma^{\prime}$ instead of $\sigma$ as explained in [16]. Note that, while it is possible to use the proof of Theorem 5 to compute an upper-bound of $T$, we do not provide it because the developments carried out in the appendix are subject to some conservatism which would typically lead to a small constant.

\section{Experimental validation}

We have implemented the proposed strategy on a benchmark. The event-triggered controller is located on a remote computer which communicates with the robot via a IEEE $802.11 \mathrm{~g}$ wireless network. The state of the robot is measured by cameras which communicate with the control unit via a dedicated wired network. Because of the time-interval between each position update and the computation time, the triggering condition is periodically verified every $T_{\text {eval }}=50 \mathrm{~ms}$, and not continuously as in Section 3-4. Experimental results show that the pe$\operatorname{riod} T_{\text {eval }}$ is sufficiently small to maintain the properties ensured by Theorem 5, which is consistent with Section 4.3 .

The reduction of the usage of the communication channel only concerns the transmissions from the controller to the robot, which corresponds to the dashed channel in Figure 2 (and not from the robot to the controller where periodic sampling is applied). The controller parameters in $(7)$ are $c_{1}=30, c_{2}=0.8, c_{3}=0.9, \gamma=1.5$.

We focus on two types of reference trajectories whose paths respectively describe an ellipse and a lemniscate and which ensure the satisfaction of Assumption 3. The triggering condition (15) has been implemented with $\sigma=0.5$ and we have studied the influence of $\varepsilon$. Simulations have been run over ${ }^{2} T_{\text {simulation }}=60 \mathrm{~s}$. The usage of the communication network is quantified using the percentage (\# stands for 'number' below)

$$
r:=100 \times \# \text { control updates } \times \frac{T_{\text {eval }}}{T_{\text {simulation }}} .
$$

\footnotetext{
1 We actually have a periodic event-triggered controller $[6,10]$.

${ }_{2}^{2}$ Tests have shown that the results do not significantly vary for bigger values of $T_{\text {simulation }}$.
} 


\begin{tabular}{ccccc}
$\varepsilon$ & \multicolumn{2}{c}{ Ellipse } & \multicolumn{2}{c}{ Lemniscate } \\
\hline & $r$ & $e$ & $r$ & $e$ \\
\hline 0.01 & $10.4 \%$ & 0.1386 & $23.6 \%$ & 0.3476 \\
0.05 & $2.44 \%$ & 0.2476 & $10.9 \%$ & 0.3907 \\
0.1 & $2.33 \%$ & 0.2397 & $6.12 \%$ & 0.5759 \\
\hline
\end{tabular}

Table 1

Values of $r$ and $e$ for different $\varepsilon$

The performance of the controller is evaluated by the constant $e$ that is the maximum value of $\mid\left(x-x_{r}, y-\right.$ $\left.y_{r}, \theta-\theta_{r}\right) \mid$, which is (heuristically) normalised according to the dimensions of the system, over the second half of the experiment, i.e.

$$
e:=\max _{t \in\left[\frac{1}{2} T_{\text {simulation }}, T_{\text {simulation }}\right]}\left|\left(\begin{array}{c}
\left(x(t)-x_{r}(t)\right) / 0.1 \\
\left(y(t)-y_{r}(t)\right) / 0.1 \\
\theta(t)-\theta_{r}(t)
\end{array}\right)\right| .
$$

In that way, $e$ quantifies the tracking accuracy of the robot after the transient period. The scaling coefficient 0.1 in (18) approximatively corresponds to the robot diameter, i.e. $100 \mathrm{~mm}$. Table 1 shows that the controller only uses the communication channel $23.6 \%$ of the time, in the worst case, and that this quantity can be significantly decreased by varying $\varepsilon$. Thus, we see that to increase $\varepsilon$ reduces the usage of the communication channel at the price of degraded tracking performances (in general).

We then compared the event-triggered controller with a corresponding time-triggered one. The same controller (7) is considered but the control input is transmitted to the robot every $T_{\text {sampling seconds. We are not aware }}$ of any analytical result which allows to derive an upper bound on $T_{\text {sampling. Hence, we have selected } T_{\text {sampling }} \text { to }}$ be the average inter-transmission interval of the corresponding event-triggered controller. In that way, both controllers do generate the same amount of transmissions over an experiment. Figure 3 shows the average values of $e$ for different $\varepsilon$ obtained over 4 tests. We see that both controllers generate a similar tracking performance for small values of $\varepsilon$ and that the event-triggered controller outperforms the time-triggered one for large values of $\varepsilon$, i.e. when the wireless network is used less often. These results suggest that it is possible to reduce the usage of the wireless network using the event-triggered controller provided that we agree to give up a bit on the tracking accuracy.

\section{Conclusion}

An event-triggered tracking controller has been designed and implemented for a standard model of mobile robots. A global practical stability property together with the
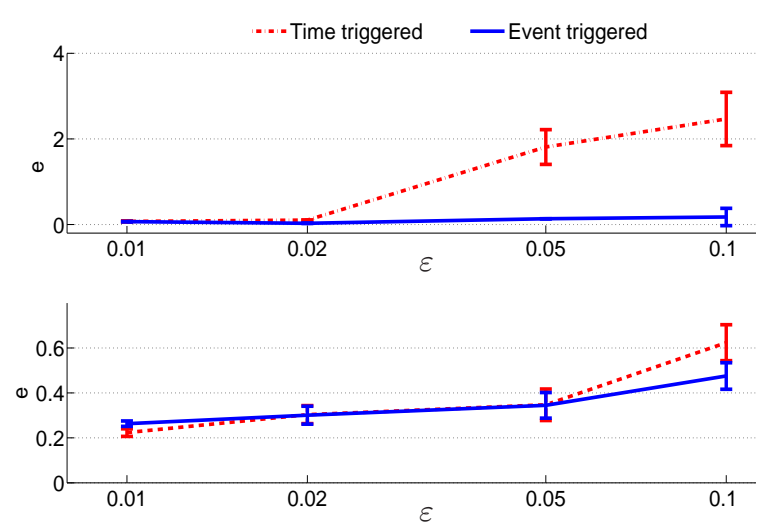

Fig. 3. Experimental results when the reference describes an ellipse (top) and a lemniscate (bottom).

existence of a strictly positive amount of time between two transmissions have been proved under appropriate conditions on the reference trajectories to be tracked. Finally, the experimental results illustrate the potential of event-triggering techniques over periodic sampling and support the on-going research on nonlinear eventtriggered control in general, as these demonstrate the benefits of the paradigm on a real system.

\section{Acknowledgements}

The authors are grateful to Lucas Bchini for his help for the experimental results reported in Section 5, and to the anonymous reviewers for their valuable comments which lead to significant improvements.

\section{A Appendix}

Proof of Lemma 4. Let $q \in \mathcal{Q}$ such that $V(q) \geq \varepsilon^{2}$. In view of the definition of $V$ in (13), the following three cases can occur: (i) $\frac{1}{2} \bar{x}_{e}^{2} \geq \frac{\varepsilon^{2}}{3}$; (ii) $\frac{1}{2 \gamma} \theta_{e}^{2} \geq \frac{\varepsilon^{2}}{3}$; (iii) $\frac{1}{2} y_{e}^{2} \geq \frac{\varepsilon^{2}}{3}$. Since $\Sigma(q)=c_{1} \bar{x}_{e}^{2}+c_{2} \theta_{e}^{2}+c_{3} w^{2} y_{e}^{2}$, (16) holds with $\vartheta=\frac{2}{3} \varepsilon^{2} \min \left\{c_{1}, c_{2} \gamma\right\}$ for cases (i) and (ii). Case (iii) requires more attention. Suppose that $\theta_{e}^{2} \leq \bar{\varepsilon}^{2}$ with $\bar{\varepsilon}>0$ sufficiently small such that $\left(\frac{2}{3}\right)^{\frac{1}{2}} \varepsilon c\left|\operatorname{sinc} \theta_{e}\right|-$ $2 c_{2}\left|\theta_{e}\right|>\frac{1}{2}\left(\frac{2}{3}\right)^{\frac{1}{2}} \varepsilon c$ (otherwise $\theta_{e}^{2} \geq \bar{\varepsilon}^{2}$ and (16) holds with $\left.\vartheta=c_{2} \bar{\varepsilon}^{2}\right)$. We want to ensure $w^{2} \geq 1$ by appropriately selecting $\gamma$. Since $w=w_{r}+\gamma\left(y_{e} v_{r} \operatorname{sinc} \theta_{e}+c_{2} \theta_{e}\right)$, $w^{2}$ is a polynomial in $\gamma$. The idea is to first prove that the leading term $\left(y_{e} v_{r} \operatorname{sinc} \theta_{e}+c_{2} \theta_{e}\right)^{2}$ is bigger than a constant $\lambda>0$. We can then select $\gamma$ sufficiently large to obtain that $w^{2} \geq 1$. It holds that, in view of Assumption 
3 and since we are in case (iii),

$$
\begin{gathered}
\left(y_{e} v_{r} \operatorname{sinc} \theta_{e}+c_{2} \theta_{e}\right)^{2} \geq y_{e}^{2} v_{r}^{2} \operatorname{sinc} \theta_{e}^{2}+c_{2}^{2} \theta_{e}^{2} \\
-2\left|y_{e} v_{r} \operatorname{sinc} \theta_{e}\right| c_{2}\left|\theta_{e}\right| \\
\geq\left|y_{e} v_{r} \operatorname{sinc} \theta_{e}\right|\left(\left|y_{e} v_{r} \operatorname{sinc} \theta_{e}\right|-2 c_{2}\left|\theta_{e}\right|\right) \\
\geq\left(\frac{2}{3}\right)^{\frac{1}{2}} \varepsilon c\left|\operatorname{sinc} \theta_{e}\right|\left(\left(\frac{2}{3}\right)^{\frac{1}{2}} \varepsilon c\left|\operatorname{sinc} \theta_{e}\right|-2 c_{2}\left|\theta_{e}\right|\right) .
\end{gathered}
$$

In view of the conditions assumed on $\theta_{e},\left(y_{e} v_{r} \operatorname{sinc} \theta_{e}+\right.$ $\left.c_{2} \theta_{e}\right)^{2} \geq \lambda$ for some $\lambda>0$. Consequently, for $w_{r}$ such that $\left|w_{r}\right| \leq M$ (which is the case in our study according to Assumption 3),

$$
\begin{aligned}
w^{2} & \geq \gamma^{2}\left(y_{e} v_{r} \operatorname{sinc} \theta_{e}+c_{2} \theta_{e}\right)^{2}-2\left|w_{r}\right| \gamma\left|y_{e} v_{r} \operatorname{sinc} \theta_{e}+c_{2} \theta_{e}\right| \\
& \geq \gamma^{2}\left|y_{e} v_{r} \operatorname{sinc} \theta_{e}+c_{2} \theta_{e}\right| \sqrt{\lambda}-2 M \gamma\left|y_{e} v_{r} \operatorname{sinc} \theta_{e}+c_{2} \theta_{e}\right|
\end{aligned}
$$

It suffices to select $\gamma$ sufficiently large for the right handside of the last inequality above to be bigger than 1 (using the fact that $\left.\left|y_{e} v_{r} \operatorname{sinc} \theta_{e}+c_{2} \theta_{e}\right| \geq \sqrt{\lambda}\right)$. We have proved that (16) holds with $\vartheta=\frac{2}{3} \varepsilon^{2} \min \left\{c_{1}, c_{2} \gamma, c_{3}, c_{2} \bar{\varepsilon}^{2}\right\}$.

Proof of Theorem 5. Let $c_{1}, c_{2}, c_{3}, \varepsilon>0$ and $\gamma>0$ be such that (16) holds. Let $q \in C$. According to the definition of the set $C, V(q)>\varepsilon^{2}$ implies $\langle\nabla V(q), f(q)\rangle \leq$ $-\sigma \Sigma(q)$, which leads to, in view of Lemma 4 ,

$$
\langle\nabla V(q), f(q)\rangle \leq-\sigma \vartheta<0
$$

On the other hand, it holds that, for any $q \in D$,

$$
V(g(q)) \leq V(q)
$$

Boundedness of the solutions. We now derive a boundedness property for the solutions to (11) based on (A.3) and (A.4), which will be used to prove that any maximal solution is complete and that the solutions have a uniform semiglobal dwell-time. Let $\Delta>0$ and $\phi=\left(\phi_{x_{e}}, \phi_{y_{e}}, \phi_{\theta_{e}}, \phi_{\hat{w}}, \phi_{\hat{v}}, \phi_{\tau}\right)$ be a solution to (11) with $|\phi(0,0)| \leq \Delta$. Denote $\phi_{\bar{x}_{e}}:=\phi_{x_{e}}-c_{3} w \phi_{y_{e}}$. We want to upper bound $\left|\phi_{\bar{x}_{e}}(0,0)\right|$ first. From (7), it holds that

$$
\begin{aligned}
|w(0,0)| \leq & \left|w_{r}(0,0)\right|+\gamma\left|\phi_{y_{e}}(0,0)\right|\left|v_{r}(0,0)\right| \\
& \times\left|\operatorname{sinc}\left(\phi_{\theta_{e}}(0,0)\right)\right|+c_{2} \gamma\left|\phi_{\theta_{e}}(0,0)\right| .
\end{aligned}
$$

Using Assumption 3 and $\operatorname{sinc}(z) \leq 1$ for any $z \in \mathbb{R}$, $|w(0,0)| \leq M+\gamma \Delta M+c_{2} \gamma \Delta=: \bar{\Delta}_{w}$. Since $\phi_{\bar{x}_{e}}(0,0)=$ $\phi_{x_{e}}(0,0)-c_{3} w \phi_{y_{e}}(0,0),\left|\phi_{\bar{x}_{e}}(0,0)\right| \leq\left|\phi_{x_{e}}(0,0)\right|+$ $c_{3}|w|\left|\phi_{y_{e}}(0,0)\right| \leq \Delta+c_{3} \Delta_{w} \Delta=\left(1+c_{3} \Delta_{w}\right) \Delta$. Therefore

$$
\begin{aligned}
\left|\left(\phi_{\bar{x}_{e}}(0,0), \phi_{\theta_{e}}(0,0), \phi_{y_{e}}(0,0)\right)\right| \\
\quad \leq\left|\phi_{\bar{x}_{e}}(0,0)\right|+\left|\left(\phi_{\theta_{e}}(0,0), \phi_{y_{e}}(0,0)\right)\right| \\
\quad \leq\left(1+c_{3} \Delta_{w}\right) \Delta+\Delta=\left(2+c_{3} \Delta_{w}\right) \Delta
\end{aligned}
$$

Note that $\underline{\gamma}\left|\left(\bar{x}_{e}, \theta_{e}, y_{e}\right)\right|^{2} \leq V(q) \leq \bar{\gamma}\left|\left(\bar{x}_{e}, \theta_{e}, y_{e}\right)\right|^{2}$ with $\underline{\gamma}=\frac{1}{2} \min \left\{1, \frac{1}{\gamma}\right\}$ and $\bar{\gamma}=\frac{1}{2} \max \left\{1, \frac{1}{\gamma}\right\}$ for any $q \in \mathcal{Q}$. In that way, we derive from (A.3), (A.4) and (A.6) that, for $(t, j) \in \operatorname{dom} \phi$,

$$
\begin{aligned}
V(\phi(t, j)) & \leq \max \left\{V(\phi(0,0)), \varepsilon^{2}\right\} \\
& \leq \max \left\{\bar{\gamma}\left|\left(\phi_{\bar{x}_{e}}(0,0), \phi_{\theta_{e}}(0,0), \phi_{y_{e}}(0,0)\right)\right|^{2}, \varepsilon^{2}\right\} \\
& \leq \max \left\{\bar{\gamma}\left(2+c_{3} \Delta_{w}\right)^{2} \Delta^{2}, \varepsilon^{2}\right\}
\end{aligned}
$$

from which we obtain $\left|\left(\phi_{\bar{x}_{e}}(t, j), \phi_{y_{e}}(t, j), \phi_{\theta_{e}}(t, j)\right)\right| \leq$ $\Delta_{V}$ for some $\Delta_{V}>0$ which only depends on the controller parameters, $\Delta, M$ and $\varepsilon$. As a consequence, using Assumption 3, we deduce that $w(t, j)$ and $v(t, j)$ are bounded by a constant $\Delta_{v, w}>0$ which depends on the same constants as $\Delta_{V}$, for any $(t, j) \in \operatorname{dom} \phi$ in view of (7). In view of $(12),\left|\phi_{\hat{w}}(t, j)\right| \leq \Delta_{w, v}$ for $(t, j) \in \operatorname{dom} \phi$ with $j \geq 1$ and $\left|\phi_{\hat{w}}(0,0)\right| \leq \Delta$ (since $\left.|\phi(0,0)| \leq \Delta\right)$, we derive that $\phi_{\hat{w}}$ is bounded by $\Delta_{v, w}+\Delta$. We similarly deduce that $\left|\phi_{\hat{v}}(t, j)\right| \leq \Delta_{v, w}+\Delta$ for any $(t, j) \in \operatorname{dom} \phi$. We have shown that for all $(t, j) \in \operatorname{dom} \phi$

$$
\left|\left(\phi_{x_{e}}(t, j), \phi_{y_{e}}(t, j), \phi_{\theta_{e}}(t, j), \phi_{\hat{w}}(t, j), \phi_{\hat{v}}(t, j)\right)\right| \leq \bar{\Delta}
$$

for some $\bar{\Delta}>0$ which only depends on the controller parameters, $\Delta, M$ and $\varepsilon$.

Uniform semiglobal dwell-times. We now show that the solutions to (11) have a uniform semiglobal dwell-time. Let $\Delta \geq 0, \phi$ be a solution to (11) with $|\phi(0,0)| \leq$ $\Delta,\left(t_{1}, 1\right) \in \operatorname{dom} \phi$ and $\left(t_{2}, 1\right)$ where $t_{1}=\inf I^{1}$ and $t_{2}=\sup I^{1}$ (note that if $I^{1}=\emptyset$ or $I^{1}$ is unbounded, $\phi$ jumps no more than once and the desired result holds). Necessarily $V\left(\phi\left(t_{1}, 1\right)\right) \geq \varepsilon^{2}$ as $\phi\left(t_{1}, 0\right) \in D$ and so $V\left(\phi\left(t_{1}, 0\right)\right)=V\left(\phi\left(t_{1}, 1\right)\right) \geq \varepsilon^{2}$. If there exists $t \in I^{1}$ such that $V(\phi(t, 1)) \leq \varepsilon^{2}$ then $t_{2}=\infty$ as the set $\{q$ : $\left.V(q) \leq \varepsilon^{2}\right\}$ is strongly pre-forward invariant for system (11) in view of (A.3) and (A.4), in which case (2) holds for any $\eta>0$. When for any $t \in I^{1}, V(\phi(t, 1)) \geq \varepsilon^{2}$, we deduce that $t_{2}-t_{1}$ is bigger than the time $T$ it takes for $\Lambda(\phi)$ to grow from 0 (its value at $\left.\left(t_{1}, 1\right)\right)$ to $(1-\sigma) \vartheta$ (as this constant lower bounds $(1-\sigma) \Sigma(\phi)$ when $V(\phi) \geq \varepsilon^{2}$ according to Lemma 4 ). In view of (A.8) and since $\Lambda$ is continuously differentiable with respect to $q$, we can upper bound $\left|\frac{\partial \Lambda(q)}{\partial q} f(q)\right|$ by a constant $\Delta_{\Lambda}$ which depends on $\Delta, M, \varepsilon$ and the controller parameters. Hence, $t_{2}-t_{1}$ is lower bounded by the time $T$ which verifies $\Delta_{\Lambda} T=(1-\sigma) \vartheta$, thus $T=(1-\sigma) \vartheta \Delta_{\Lambda}^{-1}>0$. Two jumps are separated by $T>0$ : (2) holds with $\eta(\Delta)=T$. Item 
(ii) of Theorem 5 follows by induction by repeating the same arguments for $t_{j+1}-t_{j}$ with $j \in \mathbb{Z}_{\geq 0}$.

Item (i) of Theorem 5. Let $r>0$ and $\varepsilon^{\prime}>\varepsilon$ and consider a solution $\phi$ to $(11)$ such that $V(\phi(0,0)) \leq r$. Let $(t, j) \in \operatorname{dom} \phi$ with $j \geq 1$ and $t_{1}=\inf I^{1}$. Since $V(\phi(0,0)) \leq r$, there exists $\Delta \geq 0$ such that $\left|\left(\phi_{x_{e}}\left(t_{1}, 1\right), \phi_{y_{e}}\left(t_{1}, 1\right), \phi_{\theta_{e}}\left(t_{1}, 1\right), \phi_{\hat{w}}\left(t_{1}, 1\right), \phi_{\hat{v}}\left(t_{1}, 1\right)\right)\right| \leq$ $\Delta$ for $\left(t_{1}, 1\right) \in \operatorname{dom} \phi$ in view of (13), Assumption 3 and (12). According to item (ii) of Theorem 5, we derive that $t-t_{1} \geq(j-1) \eta(r)$ where we write $\eta(r)$ (instead of $\eta(\Delta)$ with some abuse of notation). We notice that $V(\phi(t, j)) \leq \max \left\{V(\phi(0,0))-\sigma \vartheta t, \varepsilon^{2}\right\} \leq$ $\max \left\{r-\sigma \vartheta t, \varepsilon^{2}\right\}$ in view of (A.3) and (A.4). Consequently, $t+j \geq T^{\prime}$ with $T^{\prime}=\left(1+\eta(r)^{-1}\right)^{-1}\left(\frac{r-\varepsilon^{\prime 2}}{\sigma \vartheta}-1\right)$, implies $V(\phi(t, j)) \leq \varepsilon^{\prime 2}$. Note that when $t_{1}=\infty$, we have that $\operatorname{dom} \phi=\mathbb{R}_{\geq 0} \times\{0\}$ and we obtain $t+j=t \geq \frac{r-\varepsilon^{\prime 2}}{\sigma \vartheta}$ implies $V(\phi(t, j)) \leq \varepsilon^{\prime 2}$ for $(t, j) \in \operatorname{dom} \phi$. We have thus proved that for any $r>0$, $\varepsilon^{\prime}>\varepsilon$, there exists $\bar{T}>0$ such that for any solution $\phi$ to (11), $t+j \geq \bar{T}$ with $(t, j) \in \operatorname{dom} \phi$ implies $V(\phi(t, j)) \leq \varepsilon^{\prime 2}$.

We now use this uniform global attractivity property together with the first inequality in (A.7) to prove item (i) of Theorem 5 . We follow similar arguments as in the proof of Theorem 3.40 in [5] for that purpose. We consider $\beta_{0}(r, s)=\max \{\sup \{V(\phi(t, j)): \phi \in \mathcal{S}(\xi), V(\xi) \leq$ $\left.r, t+j \geq s\}-\varepsilon^{2}, 0\right\}+r \exp (-s)$ for $r, s \geq 0$ (where $\mathcal{S}(\bar{\xi})$ is the set of the solutions to (11) starting at $\xi$ ) which ensures $V(\phi(t, j))-\varepsilon^{2} \leq \beta_{0}(V(\phi(0,0)), t+j)$ for any solution $\phi$ to (11). The function $\beta_{0}$ is strictly increasing in $r$ and decreasing in $s$ and $\beta_{0}(r, s) \leq \max \left\{r-\varepsilon^{2}, 0\right\}+r$ in view of (A.7). Hence $\lim _{r \rightarrow 0^{+}} \beta_{0}(r, s)=0$ for each $s \geq 0$. Furthermore, $\lim _{s \rightarrow \infty} \beta(r, s)=0$ in view of the uniform global attractivity shown above. Since $\beta_{0}$ is not necessarily continuous, we use Lemma 3.41 in [5] to upper-bound it by $\beta \in \mathcal{K} \mathcal{L}$. As a consequence, item (i) of Theorem 5 holds.

Completeness of the maximal solutions Let $\phi$ be a maximal solution to (11). In view of $(12), \phi$ is nontrivial as $f(q)$ always belong to the tangent cone to $C$ at $q$ (see Definition 5.12 in [5]) for any $q \in C \backslash D$, see Proposition 6.10 in [5]. Noting that $C \cup D=\mathcal{Q}$, the only potential obstacle to the completeness of $\phi$ is if it explodes in finite-time according to Proposition 6.10 in [5], but that is not possible in view of (A.8) and the dynamics of the $\tau$-variable. Hence $\phi$ is complete.

\section{References}

[1] K.E. Arzén. A simple event-based PID controller. In Proceedings of the 14th IFAC World Congress, Beijing, China, volume 18, pages 423-428, 1999.

[2] K.J. Aström and B.M. Bernhardsson. Comparison of Riemann and Lebesgue sampling for first order stochastic systems. In IEEE Conference on Decision and Control, Las Vegas, U.S.A., pages 2011-2016, 2002.

[3] M.C.F. Donkers and W.P.M.H. Heemels. Outputbased event-triggered control with guaranteed $\mathcal{L}_{\infty}$-gain and improved and decentralized event-triggering. IEEE Transactions on Automatic Control, 57(6):1362-1376, 2012.

[4] F. Forni, S. Galeani, D. Nešić, and L. Zaccarian. Eventtriggered transmission for linear control over communication channels. Automatica, 50(2):490-498, 2014.

[5] R. Goebel, R.G. Sanfelice, and A.R. Teel. Hybrid dynamical systems. Princeton, U.S.A., 2012.

[6] W.P.M.H. Heemels, M.C.F. Donkers, and A.R. Teel. Periodic event-triggered control for linear systems. IEEE Transactions Automatic Control, 58(4):847-861, 2013.

[7] W.P.M.H. Heemels, J.H. Sandee, and P.P.J. van den Bosch. Analysis of event-driven controllers for linear systems. International Journal of Control, 81(4):571-590, 2009.

[8] Z.-P. Jiang and H. Nijmeijer. Tracking control of mobile robots: a case study in backstepping. Automatica, 33(7):1393-1399, 1997.

[9] Y. Kanayama, Y. Kimura, F. Miyazaki, and T. Noguchi. A stable tracking control method for an autonomous mobile robot. In ICRA (IEEE International Conference on Robotics and Automation), pages 384-389, 1990.

[10] R. Postoyan, A. Anta, W.P.M.H. Heemels, P. Tabuada, and D. Nešić. Periodic event-triggered control for nonlinear systems. In IEEE Conference on Decision and Control, Florence, Italy, pages 7397-7402, 2013.

[11] R. Postoyan, M.C. Bragagnolo, E. Galbrun, J. Daafouz, D. Nešić, and E.B. Castelan. Nonlinear event-triggered tracking control of a mobile robot: design, analysis and experimental results. In IFAC Symposium on Nonlinear Control, Toulouse, France, pages 318-323, 2013.

[12] R. Postoyan, P. Tabuada, D. Nešić, and A. Anta. A framework for the event-triggered stabilization of nonlinear systems. IEEE Transactions on Automatic Control (available on-line).

[13] R. Postoyan, N. van de Wouw, D. Nešić, and W.P.M.H. Heemels. Tracking control for nonlinear networked control systems. IEEE Transactions on Automatic Control, 59(6):1539-1554, 2014.

[14] C. Santos, M. Mazo Jr., and F. Espinosa. Adaptive selftriggered control of a remotely operated robot. In Advances in Autonomous Robotics, pages 61-72. Springer, 2012.

[15] A. Seuret, C. Prieur, and N. Marchand. Stability of nonlinear systems by means of event-triggered sampling algorithms. IMA Journal of Mathematical Control and Information, 31(3):415-433, 2013.

[16] P. Tabuada. Event-triggered real-time scheduling of stabilizing control tasks. IEEE Transactions on Automatic Control, 52(9):1680-1685, 2007.

[17] P. Tallapragada and N. Chopra. On event triggered tracking for nonlinear systems. IEEE Transactions on Automatic Control, 58(9):2343-2348, 2013. 